\title{
An Ontology-driven Multisensorial Platform to Enable Unobtrusive Human Monitoring and Independent Living
}

\author{
Pioggia G. ${ }^{1}$, Ricci G. ${ }^{2}$, Bonfiglio S. ${ }^{3}$, Bekiaris E. ${ }^{4}$, Siciliano G. ${ }^{2}$, De Rossi D. ${ }^{2}$ \\ ${ }^{1}$ Institute of Clinical Physiology - CNR, Pisa, Italy \\ ${ }^{2}$ University of Pisa, Italy \\ ${ }^{3}$ Philips FIMI, Milan, Italy \\ ${ }^{4}$ Centre for Research \& Technology Hellas/Hellenic Institute of Transport, Greece \\ E-mail: giovanni.pioggia@ifc.cnr.it
}

\begin{abstract}
The profound, pervasive and enduring consequences of ageing population present enormous challenges as well as enormous opportunities for Information and Communication Technology. The EU funded OASIS project, a Large Scale Integrated Project, is aimed to develop an open and innovative reference architecture, based upon ontologies and semantic services, that will allow plug and play and cost-effective interconnection of existing and new services in all domains required for the independent and autonomous living of the elderly and their enhanced quality of life. Among other technological advances, in OASIS we are developing a smart multisensorial platform for monitoring the lower limbs movements, as well as the muscular activations. We are using unobtrusive integrated sensors to transduce posture and kinematic variables and to acquire surface Electromiography (sEMG). The platform is able to analyze and merge the sEMG signals and kinematics variables to provide a single coherent dynamic information of the acquired movements. A Predictive Dynamic Model (PDM) based on machine learning techniques assesses the physiological muscular recruitments as well as muscular fatigue and physiological conditions.
\end{abstract}

Keywords: unobtrusive integrated sensors, human monitoring, predictive dynamic model

\section{Introduction}

Age and disability are strongly correlated: $15 \%$ of the EU population has a disability; $70 \%$ of them will be over 60 by 2020 . There exist two main trends in the 21 century. One is the rapid development of ICT, which has affected all areas of life of people and radically changed the way- people live and tackle many activities. People have entered into digital times. The other is the trend of aging population, which has attracted attention from government as well as business firms. Potential business opportunities exist exactly at the crossing point of these two trends.

The older population is growing at a considerably faster rate than that of the world's total population. In absolute terms, the number of older persons has tripled over the last 50 years and will more than triple again over the next 50-year period. In relative terms, the percentage of older persons is projected to more than double worldwide over the next half century, to expand by more than three times, to reach nearly 2 billion, in 2050. This rapid expansion in the older population affects practically all regions of the world and is increasing. Currently, the annual growth of the older population $(1.9 \%)$ is significantly higher than that of the total population $(1.02 \%)$.

In the near future, the difference between those two rates is expected to become even larger, as the baby boom generation starts ageing in many parts of the world, including Europe. By 2025-2030, projections indicate that the population over 60 will be growing 3.5 times as rapidly as the total population $(2.8 \%$ compared to $0.8 \%$ ). Even though the growth rate of the over $60 \mathrm{~s}$ age group is expected to decline to $1.6 \%$ in 2045-2050, it will still be more than 3 times the growth rate of the total population $(0.5 \%)$ by mid century.

\section{The OASIS Project}

OASIS is the acronym of a Large Scale Integrated Project co-financed by the European Commission (7th Framework Programme, ICT and Ageing - Grant Agreement No: 215754). The full project name is: Open architecture for Accessible Services Integration and Standardization. It started on January 1, 2008 and has a length of four years. The OASIS Consortium is 
composed of 33 Partners from 11 countries. Large Industries, SMEs, Universities, Research Centers, Non-Profit Organizations, Public Organizations and Healthcare Centers are all represented.

OASIS introduces an innovative, Ontology-driven, Open Reference Architecture and Platform, which will enable and facilitate interoperability, seamless connectivity and sharing of content between different services and ontologies in all application domains relevant to applications for the elderly and beyond. The OASIS platform (Figure 1) is open, modular, holistic, easy to use and standards abiding. It includes a set of novel tools for content/services connection and management, for user interfaces creation and adaptation and for service personalization and integration. Through this new Architecture, over 12 different types of services are connected with the OASIS Platform for the benefit of the elderly, covering user needs and wants in terms of Independent Living Applications (nutritional advisor, activity coach, brain and skills trainers, social communities platform, health monitoring and environmental control), Autonomous Mobility and Smart Workplaces Applications (elderlyfriendly transport information services, elderly-friendly route guidance, personal mobility services and smart workplace applications).

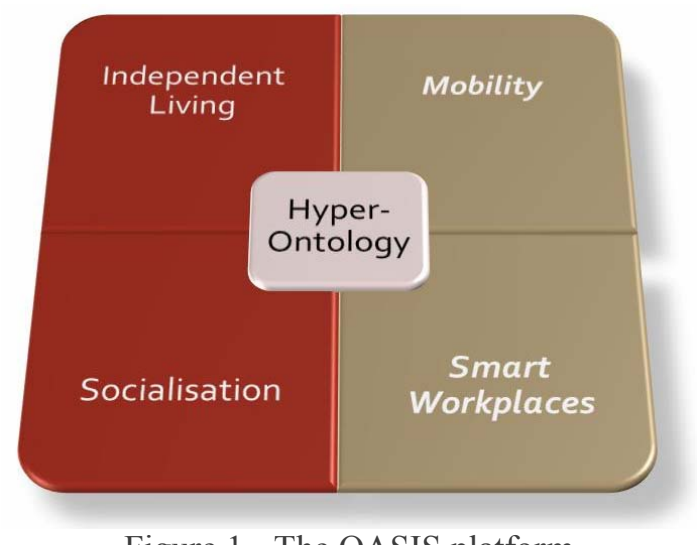

Figure 1 - The OASIS platform

Applications are all integrated as a unified, dynamic service batch, managed by the OASIS Service Centre and supporting all types of mobile devices (tablet PC, PDA, smartphone, automotive device, ITV, infokiosk, etc.) and all types of environments (living labs, sheltered homes, private homes, two car demonstrators, public transport, DSRT, etc.). As user friendliness and acceptability is a top priority for the project, a usercentred design approach is followed along the service and application development. Tested iteratively and thoroughly by hundreds of end users, their caregivers and other stakeholders, the OASIS platform and applications will be optimized and submitted for standardization by the purpose-established OASIS world-wide Industrial Forum.

\section{The Multisensorial Platform}

Presently, egocentric sensing systems consisting of biomimetic wearable suits (BWSs) for the unobtrusive biomonitoring of body-kinematics and physiological and behavioural signals are continuously improving. BWSs integrate smart sensors together with on-body signal conditioning and pre-elaboration, as well as the management of the energy consumption and wireless communication systems (Figure 3). Integrated wearable systems are able to transduce heart rate and electrocardiographic signals (ECG), as well as electromiographic signals (EMG), electrodermal response (EDR), respiratory values and arterial oxygen saturation. Acquired information is correlated to obtain blood pressure, body temperature, hearth rate variability (HRV), end tidal $\mathrm{CO} 2$ and thoracic impedance pneumographic values. A most recent breakthrough in the development of wearable systems has involved the recently developed textile substrates for the distributed on-body biochemical measurements and monitoring of body fluids. Intelligent reading strategies of unobtrusive piezoresistive networks and physiological modelling allow human upper limb kinematic variables to be inferred. This property was exploited in the realization of many sensorized garments, such as gloves, leotards, seat covers capable of reconstructing and monitoring body shape, posture and gesture to record and classify non verbal communication and body language.

The general aim of the kinesiological sEMG is the analysis of the function and coordination of muscles in different movements and postures in healthy subjects as well as in the disabled, in sedentary as well as in athletes, under laboratory conditions as well as in the field. One of the fundamental tool to measure movement is the EMG of the muscles producing the movement. Since EMG is a direct measure of alfa motor neuron activity, it provides information about the central nervous system command that generates the movement, and in case of hierarchical models of intentions of the mirror neuron system. Numerous muscle are involved in every limb movement and consequently it is necessary to record from at least two muscles with antagonistic actions. EMG can be also used as measure of force, even though relationship between EMG amplitude and force is only approximate and not linear. The timing information from the EMG signal, instead, is much more accurate. EMG data can be measured with surface (sEMG), needle or wire electrodes. sEMG technology allows information 
regarding the overall muscle function and condition to be collected from the surface of the skin. This process is non-invasive and non-painful to the subject. The sEMG signal detected on the skin surface includes information from a greater proportion of the muscle of interest than conventional clinical EMG, acquired using needle electrodes. sEMG is widely used to evaluate muscle activity and can determine which muscles are active, their degree of activity, and how active; it can also used to estimate muscle force (Figure 2 ). Furthermore, they are easy to use and provide objective inter-intra-subjects comparable data, suitable for statistical analysis. sEMG based studies have provided relevant contributions to the understanding of human movement, neuromuscular adjustments and adaptations occurring while - or following - exercise and training and, in a wider sense, to the clarification of some aspects of neural control of movement.
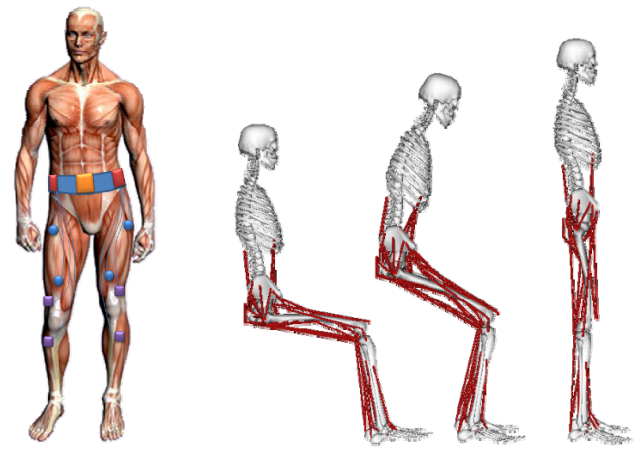

Figure 2 - Positions of sensors and a muscular model

Our aim is the ecological tracking of lower body parts motion through a $3 \mathrm{D}$ wearable motion analysis system integrated with sEMG unobtrusive wireless electrodes. We are integrating in a wireless wearable lycra garment integrated with inertial sensors and sEMG wireless probes (Figure 3), as well as strain sensing fabric and electric paths directly screen-printed for 3D motion analysis. We are using the best technologies on the marker, i.e. BTS FREEEMG for sEMG, and the sensorized lycra garment of the University of Pisa. Furthermore, we are developing a neuro-physiological model allowing movement information and muscular activations to be integrated, correlated and analysed in order to evaluate the activity planning. Furthermore we will integrate such devices within the OASIS ontology. The stream of behavioural information will be elaborated by means of intelligent and adaptive data processing techniques and artificial neural networks in order to process and code in real-time the distributed information. A parallel object-oriented framework for behavioural and sEMG features extraction, selection and discrimination will be realized.

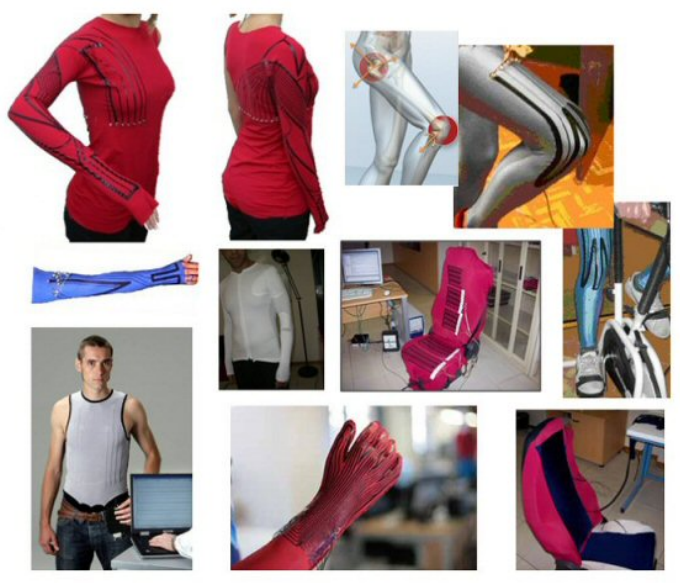

Figure 3 - Wearable sensorized garments

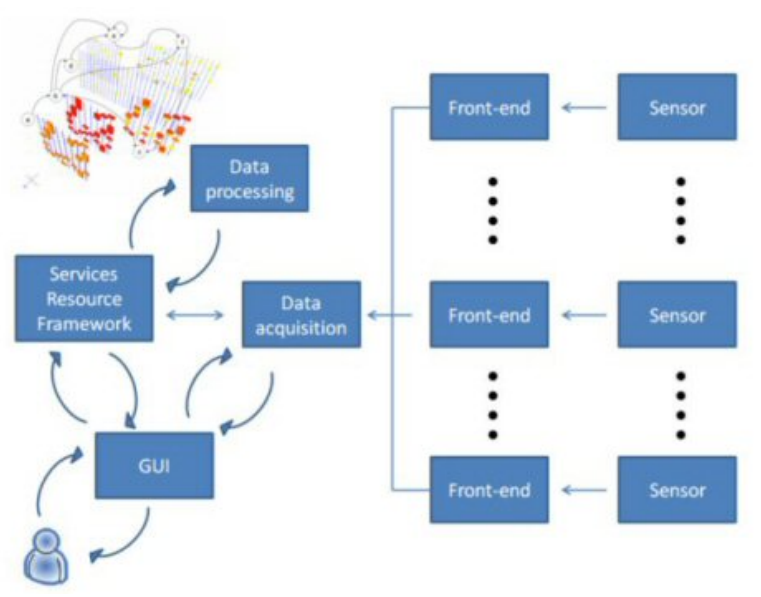

Figure 4 - Distributed computing infrastructure

Fault-tolerant mechanisms will be included in order to allow the disconnection/connection of probes without problems. Pre-processing, signal features extraction and selection, as well as classification modules will be hierarchical implemented through the design of dedicated API interfaces. Different methodologies and techniques will be parallelized and results will be standardized through a message-passing interface library.

We will develop a distributed computing infrastructure, allowing to share, select and couple each sensory patch, thus allowing them to be used as a single, unified resource. A key requirement of such an infrastructure is the efficient management and transfer of amounts of data within the multisensorial platform. A particular attention will be devoted to the definition of protocols for allowing the integration of distributed 
systems with emphasis placed on heterogeneity, scalability, and fault tolerance. A software library whose public interface following the protocol supplied by the BioSec API that uses the open source BioAPI layer will be developed in order to realise an interface able to ensure data security and ethical issues easily employed in different application scenarios.

A front-end device for the signal conditioning will be designed and developed for each patch in order to guarantee the flow of data between the sensing modules and the data acquisition section. A 44-pin cable will connect the sensing elements to the hardware front-end device. Since a high-impedance measurement strategy is adopted, the power consumption is quite zero resulting in a completely safe system. The front-end device imposes a reference current for each patch of the sensing patches and sends the voltage information to the data acquisition section. The data acquisition section will revolve around DAQ modules connected by a motherboards which runs dedicated drivers able to communicate via USB host or $10 / 100$ ethernet interfaces.

The processing application runs asynchronously in respect to the data acquisition process following a service-oriented approach. The interaction with this service is typically performed by means of a graphical Client Graphical User Interface (GUI), which hides all the complexity. A dedicated Services Resource Framework (SRF) will be developed in order to enable an easy interoperability with multisensory data and applications. The task allocation is performed by a dedicated server. First of all, the sensory discovery phase will obtain a check of the sensory elements present within the infrastructure. The resource discovery will be implemented by accessing an index information list, which provide aggregate information about each sensorized element. The physical location of the sensing elements is abstracted from the users and application programs, using a logical uniform resource identifier (URI) string to uniquely identify the interface. Fault-tolerant mechanisms will be included in order to allow the disconnection of patches without problems. Once the GUI will automatically start, the user defines the computational tasks to be executed. This information are specified in eXtensible Markup Language (XML) language.

From the client side, each sensing element transparently appears as a single device able to perform data capture. The GUI implements the recording protocol and interacts with the human subject. The result of the capture process includes the claiming subject identifier, the measurement date time, the measurement quality level, and the array of the steady state value of the signals acquired from each sensing patch.
We employ a strategy based on allocating all the data classification modules involved in the processes of activities classification, as well as FAPs identification, will be distributed among the infrastructure following a row-wise block-striped distribution. Different machine learning techniques, such as Minimum Distance Classifer (MDC), Support Vector Machine (SVM), Principal Component Analysis (PCA), Probabilistic Neural Networks (PNN), Multi-Layer Perceptron (MLP) and Kohonen Self Organizing Map (KSOM), are under implementation and testing.

\section{BIBLIOGRAPHY}

1. Pioggia G., Ferro M., Zupone G., Chirulli L., De Rossi D. Development of a Sensing Seat for Human Authentication in Proceedings of the 3rd IET International Conference on Intelligent Environments (IE07) Ulm, (Germany), 2007.

2. Ferro M., Pioggia G., Tognetti A., Carbonaro N., De Rossi D., A Sensing Seat for Human Authentication, IEEE Transactions on Information Forensics and Security, in press.

3. Lorussi F., Rocchia W., Scilingo E., Tognetti A., De Rossi D., Wearable Redundant Fabric-Based Sensors Arrays for reconstruction of Body Segment posture, IEEE Sensors Journal, 4(6), 807-818, 2004;

4. Lorussi F., Scilingo E.P., Tesconi M., Tognetti A., De Rossi D., Strain Sensing Fabric for Hand Posture and Gesture Monitoring, IEEE Transactions on Information Technology in Biomedicine, 9(3), 372381, 2005.

5. Pioggia G., Ferro M., Di Francesco F., Ahluwalia A., De Rossi D. Assessment of Bioinspired Models for Pattern Recognition in Biomimetic Systems, Bioinspiration and Biomimetics, 3(1), 016004, 2008. 